\title{
Efficacy of Laparoscopic Approach for Appendicectomy and Orchiopexy across the Pediatric Age and Size Spectrum
}

\author{
Narinder Teckchandani', Minu Bajpai ${ }^{*}$, Dalim Kumar Baidya ${ }^{2}$ \\ ${ }^{1}$ Department of Pediatric Surgery, All India Institute of Medical Sciences, Delhi, India \\ ${ }^{2}$ Department of Anaesthesiology, All India Institute of Medical Sciences, Delhi, India \\ Email: bajpai2@hotmail.com
}

Received 30 July 2014; revised 25 August 2014; accepted 17 September 2014

Copyright @ 2014 by authors and Scientific Research Publishing Inc.

This work is licensed under the Creative Commons Attribution International License (CC BY). http://creativecommons.org/licenses/by/4.0/

(c) (i) Open Access

\begin{abstract}
Context: It remains, as yet, an issue unsettled by evidence that whether the younger age and smaller size of the pediatricpatient affect the efficacy of laparoscopy for appendicectomy and intraabdominal testes. Aims: To prospectively assess the level of difficulty, surgical stress and outcomes of two commonly done laparoscopic procedures i.e., appendicectomy and orchiopexy for intraabdominal testes, across the pediatric age and size spectrum. Settings and Design: Prospective interventional study conducted in Pediatric Surgery department of a tertiary hospital. Subjects and Methods: From April 2013 to August 2014, 60 children underwent either laparoscopic interval appendicectomy or orchiopexy (unilateral or bilateral) by same surgeon. Difficulty level, metabolic stress and other clinical outcomes of the laparoscopic procedures were compared across various age groups. Statistical Analysis: Kruskal-Wallis test was used to compare various age groups with respect to the study variables. Results: The patients were divided into three groups with age ranges of 1 - 6 years, 6 - 9 years and 9 - 13 years. The three groups were very different $(P=$ 0.000) with respect to the body surface area of included children. Each of the three age groups had a similar set of 20 laparoscopic procedures viz., 10 appendicectomies, 8 unilateral orchiopexies and 2 bilateral orchiopexies. The three groups had no difference in the total level of difficulty score, duration of capnoperitoneum, postop increase in serum CRP and blood glucose levels (surrogates for metabolic stress sustained due to surgery), time to full orals and postop hospital stay. Conclusion: The younger age and smaller size of patient do not affect the efficacy of laparoscopic appendicectomy and orchiopexy.
\end{abstract}

\section{Keywords}

Pediatric Laparoscopy, Surgical Stress, Appendicectomy, Orchiopexy

\footnotetext{
${ }^{*}$ Corresponding author.
}

How to cite this paper: Teckchandani, N., Bajpai, M. and Baidya, D.K. (2014) Efficacy of Laparoscopic Approach for Appendicectomy and Orchiopexy across the Pediatric Age and Size Spectrum. Open Journal of Pediatrics, 4, 247-251. 


\section{Introduction}

Laparoscopy is an increasingly popular surgical approach for appendicectomy and intraabdominal testis in children [1]. However, it remains, as yet, an issue unsettled by evidence that whether the age and size of the patient affect the efficacy of laparoscopic approach for these procedures in younger and smaller children [2]. Due to the lack of evidence to the contrary, many pediatric surgeons may deprive the smaller children of the benefits of minimal access approach [2]. Thus, this study was designed to prospectively assess the level of difficulty, surgical stress and outcomes of two commonly done laparoscopic procedures i.e., appendicectomy and orchiopexy for intraabdominal testes, across the pediatric age and size (quantitated as body surface area) spectrum.

\section{Subjects and Methods}

A three-pronged assessment (Figure 1) of the efficacy of laparoscopic approach was done. Surgeon-perceived difficulty level, patient-sustained metabolic stress and clinical outcomes (need for conversion to open procedure, duration of capnoperitoneum, need for blood transfusion, time to full oral feeds, and length of hospital stay) of the laparoscopic approach for appendicectomy and intraabdominal testes were compared across the pediatric age and size spectrum. The study was approved by the institute ethics committee vide letter No. IESC/T-105/01.03. 2013.

From April 2013 to August 2014, 60 patients, aged upto 13 years and undergoing either laparoscopic interval appendicectomy or laparoscopic orchiopexy for intraabdominal testes (unilateral or bilateral) were prospectively recruited into this study after obtaining written, informed parental consent. None of the patients undergoing interval appendicectomy had clinical or ultrasound evidence of any residual phlegmon at the time of surgery. All laparoscopic procedures were performed by the same senior faculty pediatric surgeon (MB) to eliminate the confounding influence of varying surgical skills.

\subsection{Level of Difficulty}

Level of difficulty of the procedure was assessed as the sum of the following subjectively assigned scores by the operating surgeon:

1) Difficulty in port placement-scored from 1 ("no difficulties”) to 4 ("most difficult”).

2) Difficulty in exposure of the organ of interest (appendix or testis)-scored from 1 ("no difficulties") to 4 ("most difficult").

3) Difficulty in dissection-scored from 1 ("no difficulties") to 4 ("most difficult with need for conversion to open procedure”).

4) Difficulty in retrieval of the organ of interest (appendix or testis)-scored from 1 ("no difficulties") to 4 (“most difficult”).

\subsection{Assessment of Metabolic Stress}

Assessment of metabolic stress sustained by the patient was done by measuring the increase in serum C-reactive protein (CRP) and blood glucose levels postoperatively. Venous blood samples were taken for estimation of serum CRP and blood glucose levels before surgery and 12 hours after surgery. This timing of postoperative blood sampling was chosen on the basis of the data found in the literature showing that both-peak serum CRP levels and peak hyperglycaemic response-occur at 12 hours postop [3]-[5]. The means of postoperative increase in serum CRP and blood glucose levels were statistically compared across different age groups for significance. The

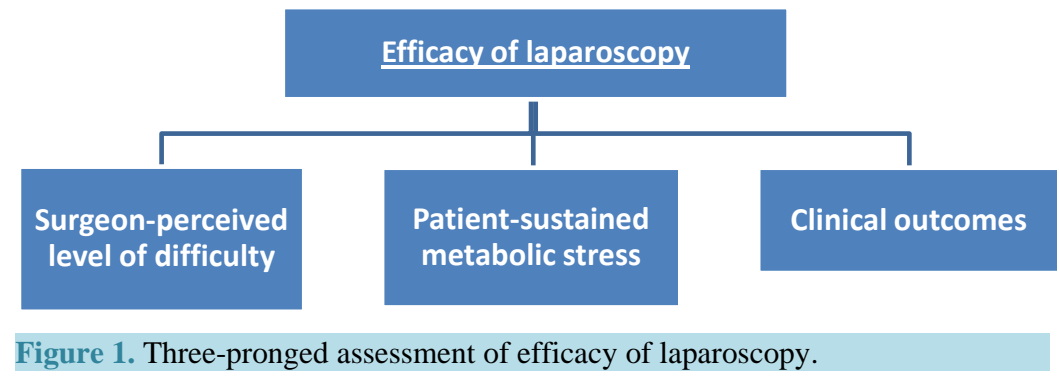


intravenous fluid being given at the time of preoperative as well as postoperative blood sampling was Isolyte-P in all patients.

The anaesthesia protocol was standardised to control for its effects on the metabolic stress assessment. In all children, general anaesthesia was induced with $2 \mu \mathrm{g} / \mathrm{kg}$ fentanyl, 2 - $3 \mathrm{mg} / \mathrm{kg}$ propofol and $0.5 \mathrm{mg} / \mathrm{kg}$ atracurium. Anaesthesia was maintained with a $\mathrm{FiO}_{2}$ of 0.5 , isoflurane and intermittent doses of fentanyl and atracurium. Intraoperatively, further doses of $0.5 \mu \mathrm{g} / \mathrm{kg}$ fentanyl were given if there occurred a more than $20 \%$ increase in heart rate and/or blood pressure above the baseline. Intraop IV fluid was Ringer lactate (RL) @ 5 ml/kg/hr; and the blood loss was additionally replaced with RL (RL: Blood: 3:1 replacement). At the end of the surgery, patient was extubated following standard extubation criteria. Once the patient was fully awake, the neuromuscular blockade was adequately reversed with $50 \mu \mathrm{g} / \mathrm{kg}$ neostigmine and $10 \mu \mathrm{g} / \mathrm{kg}$ glycopyrrolate. No steroids were given prior to extubation as it would have affected the metabolic stress assessment. Intravenous acetaminophen $10 \mathrm{mg} / \mathrm{kg} 6$ hrly was given for postoperative analgesia.

\subsection{Assessment of Clinical Outcomes}

Assessment of clinical outcomes of the laparoscopic procedure was done using the following outcome measures:

1) Need for conversion to open procedure-Yes/No.

2) Duration of capnoperitoneum (in minutes).

3) Need for blood transfusion-Yes/No.

4) Time to full oral feeds (in hours).

5) Length of hospital stay (in days).

\subsection{Operative Technique}

\subsubsection{Laparoscopic Appendicectomy}

$5 \mathrm{~mm}$ umbilical port placement by Hasson's technique was followed by carbon dioxide insufflation to a maximum of 8 to $10 \mathrm{~mm} \mathrm{Hg}$. A 3-port technique was used, placing two 5-mm ports-one each in the left lower quadrant and the suprapubic site. A 5-mm laparoscope was used. The operating table was tilted to Trendelenburg position with right side raised. The mesoappendix was identified, controlled using clips or cautery, and then divided. The appendiceal base was secured using endoloops/harmonic scalpel and divided. The appendix was removed through the umbilical port with or without the use of a laparoscopic retrieval bag. When any peritoneal contamination occurred, the abdomen and pelvis was irrigated with warmed normal saline and then suctioned dry.

\subsubsection{Laparoscopic Orchiopexy}

The laparoscopic procedure was carried out with the patient in the Trendelenburg position; an umbilical 5-mm trocar was introduced under direct vision by an open Hasson's technique, and $\mathrm{CO}_{2}$-pneumoperitoneum was induced to a maximum of 8 to $10 \mathrm{~mm} \mathrm{Hg}$. The telescope was placed through the umbilical port. Two 5-mm working ports were placed in the midclavicular line at or just below the level of the umbilicus. The gubernaculum was divided with electrocautery. The testicular vessels and the vas were subsequently mobilized. Once the testicular vessels and vas had been maximally mobilized, a dartos pouch was created in the standard fashion and a long hemostat invaginated through the scrotum into the abdomen on top of the pubic symphysis medial to the inferior epigastric vessels. The testis was grasped by the hemostat at divided gubernaculum and pulled down avoiding any twists or kinks on its pedicle.

\subsection{Statistical Analysis}

Kruskal-Wallis test was used for comparison of various variables among the three age groups and P-value < 0.05 was considered significant.

\section{Results}

Of the 60 patients, aged 1 - 13 years, 30 underwent interval appendicectomy, 24 unilateral orchiopexy and 6 had bilateral orchiopexy done laparoscopically. These patients were divided into 3 groups with age ranges of $1-6$ years, 6 - 9 years and $9-13$ years, respectively. The three groups were very significantly different $(P=0.000)$ 
with respect to the body surface area of included children. Each of these 3 age groups had undergone an equal number (total of 20 each) of appendicectomy (10 each), unilateral orchiopexy (8 each) and bilateral orchiopexy (2 each) laparoscopic procedures (Table 1), thus making them valid for a statistical comparison with respect to the level of difficulty scores, metabolic stress and clinical outcomes. Kruskal-Wallis test was used for comparison of various variables among the three age groups and P-value $<0.05$ was considered significant (Table 2).

There was no conversion of laparoscopy to open procedure in any patient. No patient required any blood transfusion.

The three age and size groups of pediatric patients undergoing similar set of laparoscopic procedures had no difference with respect to the total surgeon-perceived level of difficulty score $(\mathrm{P}=0.815)$, duration of capnoperitoneum ( $\mathrm{P}=0.414)$, postop increase in serum CRP $(\mathrm{P}=0.517)$ and blood glucose $(\mathrm{P}=0.988)$ levels, time to full orals $(\mathrm{P}=0.795)$ and postop hospital stay $(\mathrm{P}=0.679)$.

\section{Discussion}

The merits and demerits of laparoscopy in children are often debated [1] [2]. In infants and small children, the surface area for access is small, the liver margin is below the rib cage, the bladder is largely an intraabdominal structure and the abdominal cavity is small. The later-on obliterated structures, umbilical vein and arteries, and urachus remain relatively large and partially patent in infants. These anatomical characteristics make laparoscopic access and manipulation in the younger age group a more demanding and difficult task when compared to older children or adults. The article by Kravarusic [6] published in 2006 highlighted the slow adoption of minimal access surgery by pediatric surgeons and the scarcity of pediatric minimal access surgery training programs even in developed countries.

On the other hand, young children have thin and compliant abdominal wall (facilitates laparoscopic port placement and lifting of the abdominal wall with a low-pressure capnoperitoneum), well-defined anatomical landmarks and surgical planes due to lack of excess fat (thus making recognition and dissection of structures a relatively easy task) and smaller caliber vessels (that are easily amenable to hemostatic endo-gadgetry). Moreover, the miniaturization of the laparoscopic instruments in recent times has made laparoscopy more adaptable to

Table 1. Distribution of laparoscopic procedures in various age groups.

\begin{tabular}{|c|c|c|c|c|}
\hline \multirow{2}{*}{ Laparoscopic procedure } & \multicolumn{3}{|c|}{ Age (in years) } & \multirow{2}{*}{ Total } \\
\hline & $1-6$ years & $6-9$ years & $9-13$ years & \\
\hline Appendicectomy & 10 & 10 & 10 & 30 \\
\hline Unilateral orchiopexy & 8 & 8 & 8 & 24 \\
\hline Bilateral orchiopexy & 2 & 2 & 2 & 6 \\
\hline Total & 20 & 20 & 20 & 60 \\
\hline
\end{tabular}

Table 2. Comparison of study variables in various age groups.

\begin{tabular}{|c|c|c|c|c|c|c|c|}
\hline \multirow{3}{*}{ Variables } & \multicolumn{7}{|c|}{ Age (in years) } \\
\hline & \multicolumn{2}{|c|}{1 - 6 years } & \multicolumn{2}{|c|}{6 - 9 years } & \multicolumn{2}{|c|}{9 - 13 years } & \multirow{2}{*}{$\begin{array}{c}\text { P-value } \\
\text { (Kruskal-Wallis test) }\end{array}$} \\
\hline & Mean & $\begin{array}{c}\text { Std. } \\
\text { deviation }\end{array}$ & Mean & $\begin{array}{c}\text { Std. } \\
\text { deviation }\end{array}$ & Mean & $\begin{array}{c}\text { Std. } \\
\text { deviation }\end{array}$ & \\
\hline Body surface area $\left(\mathrm{m}^{2}\right)$ & 0.56 & 0.10 & 0.78 & 0.24 & 1.08 & 0.13 & 0.000 \\
\hline Total difficulty score & 5.70 & 1.06 & 5.40 & 0.97 & 5.60 & 0.84 & 0.815 \\
\hline Duration of capnoperitoneum (minutes) & 49.00 & 10.75 & 52.50 & 8.58 & 49.00 & 10.22 & 0.414 \\
\hline Postop increase in serum CRP (mg/L) & 43.16 & 6.60 & 41.94 & 8.68 & 39.31 & 7.56 & 0.517 \\
\hline Postop increase in blood glucose (mg/dl) & 58.70 & 14.61 & 57.40 & 13.99 & 58.00 & 13.51 & 0.988 \\
\hline Time to full orals (hours) & 25.80 & 2.20 & 25.80 & 3.46 & 25.00 & 3.80 & 0.795 \\
\hline Postop hospital stay (days) & 2.60 & 0.52 & 2.40 & 0.52 & 2.50 & 0.53 & 0.679 \\
\hline
\end{tabular}


smaller children.

We did not find any difference in the level of difficulty, surgical stress and clinical outcomes of two commonly done laparoscopic procedures i.e., appendicectomy and orchiopexy for intraabdominal testes across the pediatric age and size spectrum.

Magnitude of postoperative increase in serum CRP levels and postoperative hyperglycemia were used to quantify the patient-sustained metabolic stress of laparoscopic procedure. CRP is known to play a pivotal role in the pathogenesis of surgical stress and can also be used to monitor the magnitude of surgical stress. Also, a hyperglycemic response proportionate to the inflicted surgical stress has been confirmed in several studies. The postoperative hyperglycemia occurs due to suppression of insulin response and catecholamine secretion following surgical trauma [3]-[5].

\section{Conclusion}

Laparoscopic approach for appendicectomy and intraabdominal testis is as efficacious in younger and smaller children as that in older and bigger children. The younger age and smaller size of the patient, per se, do not affect the efficacy of laparoscopy for these procedures.

\section{References}

[1] Yardley, L. and Kenny, S. (2010) The Scope of Pediatric Laparoscopy. Pediatrics and Child Health, 20, 232-237. http://dx.doi.org/10.1016/j.paed.2009.12.001

[2] Agarwal, P., Bagdi, R.K., Balagopal, S., Madhu, R. and Balamourougane, P. (2006) Laparoscopy in Pediatric Surgery-Our Experience at SRMC. Sri Ramachandra Journal of Medicine, 1, 24-27.

[3] Li, P., Xu, Q., Ji, Z., Gao, Y., Zhang, X., Duan, Y., et al. (2005) Comparison of Surgical Stress between Laparoscopic and Open Appendectomy in Children. Journal of Pediatric Surgery, 4, 1279-1283. http://dx.doi.org/10.1016/j.jpedsurg.2005.05.011

[4] Desborough, J.P. (2000) The Stress Response to Trauma and Surgery. British Journal of Anaesthesia, 85, 109-117. http://dx.doi.org/10.1093/bja/85.1.109

[5] Kohl, B.A. and Deutschman, C.S. (2006) The Inflammatory Response to Surgery and Trauma. Current Opinion in Critical Care, 12, 325-332. http://dx.doi.org/10.1097/01.ccx.0000235210.85073.fc

[6] Kravarusic, D. (2006) Teaching Impact in Pediatric Minimal Access Surgery: Personal Perspective from "Fellow”. Journal of Minimal Access Surgery, 2, 216-219. 
Scientific Research Publishing (SCIRP) is one of the largest Open Access journal publishers. It is currently publishing more than 200 open access, online, peer-reviewed journals covering a wide range of academic disciplines. SCIRP serves the worldwide academic communities and contributes to the progress and application of science with its publication.

Other selected journals from SCIRP are listed as below. Submit your manuscript to us via either submit@scirp.org or Online Submission Portal.
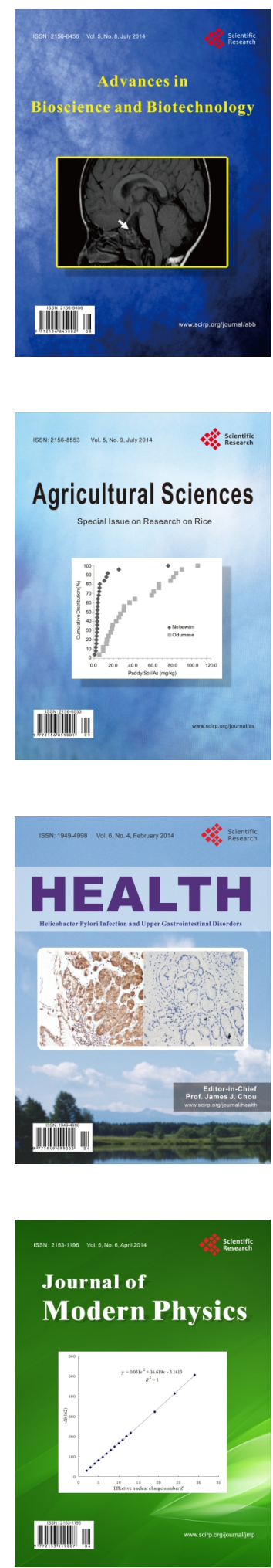
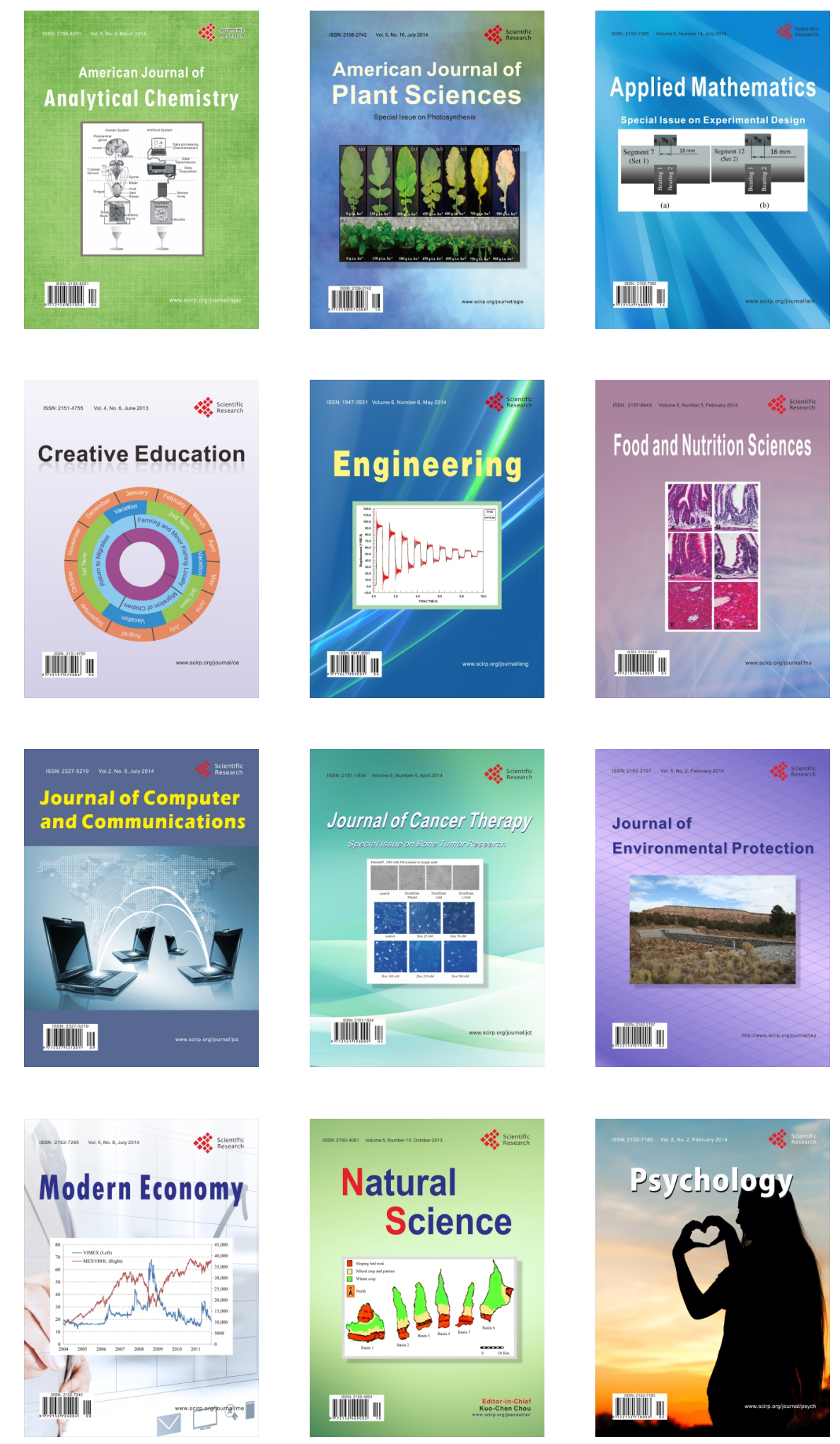